\title{
On the residual solvability of generalized free products of solvable groups
}

\author{
Delaram Kahrobaei ${ }^{2} \|$ and Stephen Majewicz $\|^{3}$ \\ ${ }^{1}$ Doctoral Program in Computer Science, Graduate Center (CUNY), New York, USA \\ ${ }^{2}$ Mathematics Department, New York City College of Technology (CUNY), New York, USA \\ ${ }^{2}$ Mathematics Department, Kingsborough Community College (CUNY), New York, USA
}

received $27^{\text {th }}$ September 2010, revised $29^{\text {th }}$ October 2010, accepted $29^{\text {th }}$ October 2010.

In this paper, we study the residual solvability of the generalized free product of solvable groups.

Keywords: residual solvability, solvable groups, amalgamated products

\section{Dedicated to Laci Babai}

\section{Introduction and Motivation}

In his celebrated paper [4], Hall introduced the residual properties. Let $\mathcal{P}$ be a property of groups, and let $G$ be a group. We say that $G$ is residually $\mathcal{P}$ if for any non-identity element $g \in G$, there exists a homomorphism $\psi$ from $G$ to a group having property $\mathcal{P}$ such that $\psi(g) \neq 1$.

We recall the definition of the generalized free products of groups. Let there be given a nonempty set of groups $\left\{G_{\lambda} \mid \lambda \in \Lambda\right\}$ together with a group $H$ which is isomorphic with a subgroup $H_{\lambda}$ of $G_{\lambda}$ by means of monomorphism $\phi_{\lambda}: H \rightarrow G_{\lambda}(\lambda \in \Lambda)$. There is an exceedingly useful object known as the free product of the $G_{\lambda}$ 's with the amalgamated subgroup $H$. Roughly speaking this is the largest group generated by the $G_{\lambda}$ 's in which the subgroups $H_{\lambda}$ are identified by means of the $\phi_{\lambda}$. Generically such groups are known as generalized free products (see [8]).

In the literature, residual finiteness has attracted much attention. In [2], for example, Baumslag studies the residual finiteness of generalized free products of nilpotent groups. Another study pursued by M. Sapir [9] and D. Wise [10] focuses on the residual finiteness of one-relator groups.

On the other hand, the exploration of residually solvable groups is in its early stages. One classical work on the residual solvability of positive one-relator groups is due to Baumslag (see [3]). The first author studied doubles of residually solvable groups (see [5]), as well as the residual solvability of the generalized free products of finitely generated nilpotent groups (see [6]). In [1], Arzhantseva, de la Harpe,

\footnotetext{
${ }^{\dagger}$ Email: dkahrobaei@gc. cuny.edu. Supported by a PSC CUNY Research Foundation of City University of New York and City Tech Foundation.

‡Email: smajewicz@kbcc. cuny. edu

1365-8050 @ 2012 Discrete Mathematics and Theoretical Computer Science (DMTCS), Nancy, France
} 
and the first author introduce the true prosolvable completion of a group and give several interesting open problems that motivate the importance of residually solvable groups.

In this paper, we generalize some of the results in [6] which carry over when nilpotent groups are replaced by solvable groups.

\section{Results}

In this section, we state our main results. Their proofs will be presented in Section 4

We begin by proving that the generalized free product of a nilpotent group and a solvable group is not necessarily perfect.

Theorem 1 The generalized free product of a nilpotent group and a solvable group amalgamated by a proper subgroup of them is not perfect.

Next we consider the case where the amalgamated subgroup of the generalized free product of two solvable groups is cyclic. By choosing an appropriate solvable filtration of each factor so that the generator of the amalgamated subgroup does not lie in the $n^{\text {th }}$ term of the derived series, it follows that the resulting amalgam is residually solvable.

Theorem 2 The generalized free product of two solvable groups amalgamating a cyclic subgroup is residually solvable.

In [6], Kahrobaei proved the generalized free product of two finitely generated solvable groups amalgamated by central subgroups is a solvable extension of a residually solvable group and, hence, is residually solvable. We generalize this result for any finite number of solvable groups.

Theorem 3 The generalized free product of a finite number of solvable groups, amalgamating a central subgroup in each of the factors, is residually solvable.

Our next theorem deals with the generalization of doubles (recall that a double is the amalgamated product of two groups whose factors are isomorphic and the amalgamated subgroups are identified under the same isomorphism).

Theorem 4 Let $\left\{A_{i} \mid i \in I\right\}$ be an arbitrary indexed family of isomorphic solvable groups such that $\bigcap_{i \in I} A_{i}=C$, and let $G$ be the generalized free product of the $A_{i}$ 's amalgamated by $C$. Then $G$ is residually solvable.

Our final result pertains to generalized free products containing a finitely generated torsion-free abelian factor.

Theorem 5 The generalized free product of a finitely generated torsion-free abelian group and a solvable group is residually solvable.

Note that Theorem 5 is a special case where the amalgamated subgroup is central in only one of the factors. 


\section{Background and Preliminary Results}

In this section, we present some preliminary material which will be needed for the proofs of our results. We begin with a theorem due to Neumann [7] which plays a crucial role in our work.

Theorem 6 (Neumann) If $K$ is a subgroup of $G=\{A * B ; C\}$, then $K$ is an HNN-extension of a tree product in which the vertex groups are conjugates of subgroups of either $A$ or $B$ and the edge groups are conjugates of subgroups of $C$. The associated subgroups involved in the HNN-extension are also conjugates of subgroups of $C$. If $K \cap A=\{1\}=K \cap B$, then $K$ is free. If $K \cap C=\{1\}$, then $K=\prod_{i \in I}{ }^{*} X_{i} * F$, where the $X_{i}$ are conjugates of subgroups of $A$ and $B$ and $F$ is free.

The next result is used in the proof of Theorem 1 . If $G$ is any group, let $G_{\mathrm{ab}}$ denote the abelianization of $G$.

Lemma 7 (Kahrobaei [6]) Let $G$ be the amalgamated product of $A$ and $B$ with $C_{A}$ and $C_{B}$ identified,

$$
G=\left\{A * B ; C_{A}=C_{B}\right\} .
$$

Then $G_{\mathrm{ab}}$ maps onto

$$
D=A_{\mathrm{ab}} / g p\left(\bar{c}_{a} \mid c_{a} \in C_{A}\right) \times B_{\mathrm{ab}} / g p\left(\bar{c}_{b}^{-1} \mid c_{b} \in C_{B}\right),
$$

where $\bar{c}_{a}$ and $\bar{c}_{b}$ are the images of $c_{a}$ and $c_{b}$ in $A_{\mathrm{ab}}$ and $B_{\mathrm{ab}}$, respectively.

Theorem 1 also relies on the next well-known theorem about the Frattini subgroup $\Phi(G)$ of a nilpotent group $G$. Let $\delta_{i} G$ denote the $i^{t h}$ derived subgroup of a group $G$.

Theorem 8 (Hirsch) If $G$ is a nilpotent group, then $\delta_{2} G \leq \Phi(G)$.

The proof of Theorem 3 makes use of the generalized central product of a set of groups. Let $Z(G)$ denote the center of a group $G$.

Definition 9 Suppose that

$$
\left\{A_{i}=\left\langle X_{i} ; R_{i}\right\rangle \mid i \in I\right\}
$$

is an indexed family of presentations, and let $C$ be a group equipped with monomorphisms

$$
\phi_{i}: C \rightarrow A_{i} \text { and } C \leq Z\left(A_{i}\right)(\text { for all } i \in I) .
$$

The group A defined by the presentation

$$
A=\left\langle\cup X_{i} ; \cup R_{i} \cup\left\{c \phi_{i} c^{-1} \phi_{j} \mid c \in C, i, j \in I\right\} \cup\left\{\left[x_{i}, x_{j}\right]=1, i, j \in I\right\}\right\rangle,
$$

where we assume that the $X_{i}$ are disjoint, is termed the generalized central product of the $A_{i}$ amalgamating the central subgroup $C$. Thus,

$$
A=\prod_{i \in I}^{\times}\left\{A_{i} ; C\right\} .
$$


It is not hard to show that

$$
A=\left(\prod_{i \in I}^{\times} A_{i}\right) / g p\left(c \phi_{i} c^{-1} \phi_{j} \mid i, j \in I, c \in C\right) .
$$

By a theorem of von Dyck, there are canonical homomorphisms $\mu_{i}: A_{i} \rightarrow A$.

Lemma 10 Each homomorphism $\mu_{i}$ is a monomorphism.

We note that the generalized central product of finitely many solvable groups is solvable. For the case of abelian factors, the generalized central product of an arbitrary number of abelian groups is abelian.

A key ingredient for proving Theorem 6 is the following:

Lemma 11 (Kahrobaei [6]) Suppose $\phi: A \rightarrow B$ is an isomorphism between two groups $A$ and B. Let $C<A$, and let $D$ be the amalgamated product of $A$ and $B$ amalgamating $C$ with $C \phi$ :

$$
D=\{A * B ; C=C \phi\}
$$

There exists a homomorphism $\psi$ from D onto one of the factors with kernel

$$
g p\left(a(a \phi)^{-1} \mid a \in A\right),
$$

and $\psi$ injects into each factor.

\section{Proofs of Main Results}

Proof of Theorem 1: Let $A$ be a nilpotent group, and let $B$ be a solvable group. Suppose $C_{A}$ and $C_{B}$ are proper subgroups of $A$ and $B$, respectively. We want to show that $G=\left\{A * B ; C_{A}=C_{B}\right\}$ is not perfect.

For any $c_{a} \in C_{A}$ and $c_{b} \in C_{B}$, let $\bar{c}_{a}$ and $\bar{c}_{b}$ denote their images in $A_{\mathrm{ab}}$ and $B_{\mathrm{ab}}$, respectively. Set $\bar{C}_{A}=g p\left(\bar{c}_{a} \mid c_{a} \in C_{A}\right)$ and $\bar{C}_{B}=g p\left(\bar{c}_{b}{ }^{-1} \mid c_{b} \in C_{B}\right)$. By Lemma 7 . $G_{\text {ab }}$ maps onto

$$
D=A_{\mathrm{ab}} / \bar{C}_{A} \times B_{\mathrm{ab}} / \bar{C}_{B} .
$$

We claim that $A_{\mathrm{ab}} / \bar{C}_{A} \neq\{1\}$. By Theorem $8, g p\left(C_{A}, \delta_{2} A\right)$ is a proper subgroup of $A$ since $C_{A}$ is. Thus,

$$
A_{\mathrm{ab}} / \bar{C}_{A} \simeq\left(A / \delta_{2} A\right) /\left(g p\left(C_{A}, \delta_{2} A\right) / \delta_{2} A\right) \simeq A / g p\left(C_{A}, \delta_{2} A\right) \nsucceq\{1\},
$$

proving our claim. It follows that $D \neq\{1\}$ and, hence, $G_{\mathrm{ab}} \neq\{1\}$. Therefore, $G$ is not perfect. 
Proof of Theorem 2; Suppose $A=g p\left(a_{i} \mid i \in I\right)$ and $B=g p\left(b_{j} \mid j \in J\right)$ are solvable groups, and let $a \in A$ and $b \in B$ be non-identity elements. There exists positive integers $m$ and $n$ such that $a \in \delta_{m} A \backslash \delta_{m+1} A$ and $b \in \delta_{n} B \backslash \delta_{n+1} B$. Let $G=\{A * B ; a=b\}$, and let $D$ be the central product of $A / \delta_{m+1} A$ and $B / \delta_{n+1} B$ amalgamating $a \delta_{m+1} A$ with $b \delta_{n+1} B$ :

$$
D=\left\{A / \delta_{m+1} A \times B / \delta_{n+1} B ; a \delta_{m+1} A=b \delta_{n+1} B\right\} .
$$

Let $\phi: G \rightarrow D$ be the homomorphism which maps $a_{i}$ to $\left(a_{i} \delta_{m+1} A, \delta_{n+1} B\right)(i \in I)$ and $b_{j}$ to $\left(\delta_{m+1} A, b_{j} \delta_{n+1} B\right)(j \in J)$, and let $K$ be the kernel of $\phi$. Then $K \cap C=\{1\}$, where $C=g p(a)=g p(b)$. By Theorem 6, $K$ is a free product of conjugates of subgroups of $A$ and $B$, and a free group. Thus $K$ is residually solvable and, hence, $G$ is an extension of a residually solvable group by a solvable group. Therefore, $G$ is residually solvable.

Proof of Theorem 3: Suppose that $\left\{A_{i} \mid i \in I\right\}$ is a finite indexed family of solvable groups, and let

$$
G=\left\{\prod_{i \in I}^{*} A_{i} ; C\right\}
$$

Let $S$ be the generalized central product of the $A_{i}$ :

$$
S=\left(\prod_{i \in I}^{\times} A_{i}\right) / g p\left(c \phi_{i} c^{-1} \phi_{j} \mid i, j \in I, c \in C\right),
$$

where the $\phi_{k}$ 's are as in Definition 9 For each $i \in I$, let $\pi_{i}$ denote the canonical homomorphism from $S$ to $A_{i}$. Since the $\pi_{i}$ coincide on $C$, they can be naturally extended to a homomorphism $\mu$ from $G$ into $S$. Let $K$ be the kernel of $\mu$, and observe that $G / K$ is solvable since $S$ is. Now, $\mu$ is one-to-one restricted to each factor; that is,

$$
K \cap A_{i}=\{1\} \text { for all } i \in I .
$$

By Theorem $6, K$ is free and, consequently, $G$ is a solvable extension of a free group. Therefore, $G$ is residually solvable.

Proof of Theorem 4; Let $\left\{A_{i} \mid i \in I\right\}$ be an arbitrary indexed family of isomorphic solvable groups such that $\bigcap_{i \in I} A_{i}=C$, and let $G$ be the generalized free product of the $A_{i}$ 's amalgamated by $C$. Pick an $i \in I$, and let $\phi: G \rightarrow A_{i}$ be an epimorphism with kernel $K$. Then $K$ is free because $\phi$ restricted to each factor is injective by Lemma 11 , and

$$
A_{i} \cap K=\{1\} \text { for all } i \in I .
$$

Therefore, $G$ is a solvable extension of a free group and is, thus, residually solvable. 
Proof of Theorem 5: Let $A$ be a finitely generated torsion-free abelian group, and let $B$ be a solvable group. Suppose

$$
G=\{A * B ; C\} .
$$

Since $A$ is finitely generated torsion-free abelian, $C$ is a direct factor of a subgroup $A_{1}$ of $A$ of finite index; that is, $A_{1}=C \times H$, where $\left[A: A_{1}\right]=n<\infty$. Let $\left\{a_{i}\right\}$ be a distinct set of coset representatives of $A_{1}$ in $A$; that is, $A / A_{1}=\bigcup_{i=1}^{n} a_{i} A_{1}$. There exists an epimorphism from $G$ onto $\bigcup_{i=1}^{n} a_{i} A_{1}$ with kernel

$$
K=g p_{G}\left(B, A_{1}\right)=\left\{\prod_{i=1}^{n *} B^{a_{i}} * A_{1} ; C\right\} .
$$

Now let $D$ be the normal closure of finitely many copies of $B$ in $K$ :

$$
D=g p_{K}\left(\bigcup_{i=1}^{t} B^{a_{i}}\right)=\left\{\prod_{i=1}^{t *} B^{a_{i}} ; C\right\} .
$$

By Theorem 4, $D$ is residually solvable. Consequently, $K$ is an extension of a residually solvable group by an abelian group. Therefore, $G$ is (residually solvable)-by-abelian and, hence, residually solvable.

\section{References}

[1] G. Arzhantseva, P. de la Harpe, D. Kahrobaei, Z. Sunik. The true prosoluble completion of a group: Examples and open problems Journal of Geometriae Dedicata, Springer Netherlands Vol. 124 (2007) No.1, 5-26

[2] G. Baumslag. On the residual finiteness of generalised free products of nilpotent groups. Trans. Amer. Math. Soc. 106 (1963), 193-209.

[3] G. Baumslag. Positive one-relator groups. Trans. Amer. Math. Soc. 156 (1971), 165-183.

[4] P. Hall. The splitting properties of relatively free groups. Proc. London Math. Soc. 4 (1954), $343-$ 356.

[5] D. Kahrobaei. Doubles of residually solvable groups. Aspects of Infinite Group Theory, Algebra and Discrete Mathematics, World Scientific Vol 1(2008)

[6] D. Kahrobaei. Residual Solvability of generalized free products of finitely generated nilpotent groups. Communications in Algebra, (to appear)(2010)

[7] H. Neumann. Generalized free producs with amalgamated subgroups. Amer. J. Math. 71 (1949), 491-540.

[8] D. J. S. Robinson. A course in the theory of groups, Springer-Verlag (1995)

[9] M. Sapir. Residual properties of one-relator groups, arXiv:1001.2829, (2010)

[10] D. Wise. The residual finiteness of quasipositive one-relator groups, J. London Math. Soc. 66, (2002), 334-350 